\title{
Propuesta de Sistema Web Personalizable para el Control de Reservaciones en Hoteles
}

\author{
Betzabet García-Mendoza, Carlos R. Jaimez-González \\ Departamento de Tecnologías de la Información, \\ Universidad Autónoma Metropolitana, Unidad Cuajimalpa, \\ Av. Vasco de Quiroga 4871, Col. Santa Fe Cuajimalpa, C.P. 05300, México, D.F. \\ 209363465@alumnos.cua.uam.mx, cjaimez@correo.cua.uam.mx
}

\begin{abstract}
Resumen. La planificación de viajes está dominada actualmente por los recursos en línea, tales como agencias de viajes, sitios web de opiniones de viaje y hoteles, redes sociales, entre otros. Cuando se ha decidido el lugar a visitar, el siguiente paso es buscar dónde hospedarse y dado que el primer medio de búsqueda es el Internet, no hay mejor oportunidad para los hoteles que incluir un motor de reservaciones en su sitio web, ofreciendo comodidad a sus clientes y abarcando un amplio mercado de ventas. En este artículo se presenta una propuesta de un sistema web personalizable para facilitar a administradores de hoteles pequeños el control de reservaciones de sus habitaciones, gestionando las ventas desde su propio sitio web; así como para facilitar y garantizar de inmediato las reservaciones para sus huéspedes potenciales. Adicionalmente, el sistema web será visualizado automáticamente en diferentes dispositivos móviles, siguiendo el patrón de diseño web adaptable.
\end{abstract}

Palabras Clave. Diseño Web Adaptable, Control de Reservaciones, Sistema Web Personalizable, Hoteles.

\section{$1 \quad$ Introducción}

La planificación de viajes está dominada actualmente por los recursos en línea: agencias de viajes, sitios web de opiniones de viaje, sitios web de opiniones de hoteles, redes sociales, sitios web de hoteles, etc. Por ejemplo, el $93 \%$ de los viajeros a nivel mundial dice que sus decisiones de reserva se ven influenciadas por las opiniones en línea [1]. Cuando una persona ha decidido el lugar a visitar, el siguiente paso es buscar dónde hospedarse y dado que el primer medio de búsqueda es el Internet, no hay mejor oportunidad para los hoteles que promocionar sus servicios por Internet. En la actualidad existe una amplia variedad de agencias de viajes, buscadores de hoteles y otras opciones, que permiten localizar hoteles en todo el mundo; sin embargo, la información del hotel que se muestra en estos sitios web es muy escasa y poco útil. 
Por otro lado, el $72 \%$ de los viajeros a nivel mundial afirman que la posibilidad de efectuar reservaciones a través de un dispositivo móvil es muy útil, y sólo el $25 \%$ de los alojamientos permite esta funcionalidad [1]. Es por ello que incluir un motor de reservaciones en el sitio web de un hotel es la mejor opción para los hoteles que desean vender de manera fácil y directa, ofreciendo comodidad a sus clientes y abarcando un amplio mercado de ventas: el Internet.

Los sitios web de hoteles tienen como objetivo principal ofrecer el servicio de alojamiento, además de otros servicios generales. Estos sitios se dividen en secciones, donde se describen los diferentes servicios con los que cuenta el hotel, mediante galerías fotográficas, así como secciones de contacto y solicitudes de reservación.

En este artículo se presenta una propuesta de un sistema web personalizable para facilitar a administradores de hoteles pequeños el control de reservaciones de sus habitaciones, gestionando las ventas desde su propio sitio web; así como para facilitar y garantizar de inmediato las reservaciones para sus huéspedes potenciales.

El resto del artículo está organizado de la siguiente manera. La sección 2 describe la estructura general, el tipo de información y las funcionalidades de algunos sitios web de hoteles que se visitaron. En la sección 3 se analizan tres herramientas para personalización de sitios web para hoteles. La sección 4 presenta la propuesta del sistema web que se está desarrollando, junto con la metodología y los avances que se tienen. Finalmente, en la sección 5 se presentan las conclusiones y el trabajo futuro.

\section{Estructura de Sitios Web de Hoteles}

Con el objetivo de conocer la estructura, tipo de información y funcionalidades con las que cuentan los sitios web de hoteles, se visitaron seis sitios web de hoteles ubicados en lugares turísticos de México: H1) Las cúpulas en Malinalco, Estado de México [2]; H2) La casa del laurel en Taxco, Guerrero [3]; H3) El encanto en Bernal, Querétaro [4]; H4) Hotel Malinalco en Malinalco, Estado de México [5]; H5) Casa abierta en Valle de Bravo, Estado de México [6]; y H6) Hotel Cristal en Chignahuapan, Puebla [7].

La Tabla 1 muestra un comparativo de la información que se ofrece en cada uno de los sitios web de los hoteles visitados; la paloma indica que el hotel tiene la información o sección en su sitio web, mientras que el tache indica que no la tiene. A continuación se proporciona una breve descripción de la información contenida en los sitios web de los hoteles analizados. 
Tabla 1. Información contenida en los sitios web de los hoteles analizados.

\begin{tabular}{lllllll}
\hline Información / Secciones & $H 1$ & $H 2$ & $H 3$ & $H 4$ & $H 5$ & $H 6$ \\
\hline Inicio / Acerca / Quiénes somos & $\checkmark$ & $\checkmark$ & $\checkmark$ & $\checkmark$ & $\checkmark$ & $\checkmark$ \\
Habitaciones & $\checkmark$ & $\checkmark$ & $\checkmark$ & $\checkmark$ & $\checkmark$ & $\checkmark$ \\
Reservaciones & $\checkmark$ & $\checkmark$ & $\checkmark$ & $\mathbf{x}$ & $\checkmark$ & $\checkmark$ \\
Servicios & $\checkmark$ & $\checkmark$ & $\checkmark$ & $\checkmark$ & $\checkmark$ & $\checkmark$ \\
Galería & $\checkmark$ & $\mathbf{x}$ & $\mathbf{x}$ & $\checkmark$ & $\checkmark$ & $\checkmark$ \\
Instalaciones & $\checkmark$ & $\mathbf{x}$ & $\checkmark$ & $\mathbf{x}$ & $\checkmark$ & $\mathbf{x}$ \\
Paquetes / Promociones & $\mathbf{x}$ & $\mathbf{x}$ & $\mathbf{x}$ & $\mathbf{x}$ & $\mathbf{x}$ & $\checkmark$ \\
Tarifas & $\checkmark$ & $\checkmark$ & $\checkmark$ & $\mathbf{x}$ & $\checkmark$ & $\checkmark$ \\
Contacto & $\mathbf{x}$ & $\mathbf{x}$ & $\checkmark$ & $\checkmark$ & $\checkmark$ & $\checkmark$ \\
Ubicación & $\checkmark$ & $\checkmark$ & $\checkmark$ & $\checkmark$ & $\checkmark$ & $\checkmark$ \\
Atracciones & $\checkmark$ & $\checkmark$ & $\checkmark$ & $\checkmark$ & $\mathbf{x}$ & $\checkmark$ \\
\hline
\end{tabular}

Inicio / Acerca / Quiénes somos. En esta sección se encuentra una breve descripción del hotel, donde se menciona la historia del hotel, cuántos años lleva funcionando, el número de habitaciones con que cuenta, sus servicios, atractivos turísticos, etc.

Habitaciones. En esta sección se encuentra la información de los diferentes tipos de habitaciones que ofrece el hotel, tal como fotografías y descripción de cada habitación, servicios con que cuenta cada habitación y el tipo de mobiliario que posee.

Reservaciones. Esta sección se compone de un formulario, donde el usuario introduce sus datos y los datos de la habitación que desea reservar. Se le indica al usuario que se verificará la disponibilidad y se le enviará posteriormente un correo electrónico con la confirmación de su reservación.

Servicios. En esta sección se describen los servicios con los que cuenta el hotel en general; pueden ser servicios básicos como: Internet, estacionamiento, cafetería, etc.; o servicios extras como: restaurante-bar, salón de fiestas, sala de negocios, etc.

Galería. La galería es una sección que puede ser un menú o estar incluida en alguna otra sección. En ella se muestran fotografías del hotel.

Instalaciones. La sección de instalaciones es una combinación de las secciones de servicios y galería; se muestran con imágenes las diferentes áreas del hotel.

Paquetes / Promociones. Esta sección se compone de paquetes o promociones definidos por el hotel. Los paquetes pueden contener diferentes servicios extras añadidos a la reservación de una habitación; mientras que las promociones pueden ser descuentos en las reservaciones en ciertas fechas.

Tarifas. La sección de tarifas muestra una tabla con los precios de cada tipo de habitación, los cuales pueden depender de las temporadas que tengan definidas en el hotel. También se tienen tarifas de los servicios extra que ofrece el hotel.

Contacto. Es una sección con un formulario y/o con información de contacto del hotel, tal como: dirección, teléfono y correo electrónico. 
Ubicación. En esta sección se muestra la dirección del hotel y un mapa (Google maps en varios de los casos) de ubicación del hotel.

Atracciones. La sección de atracciones contiene información de los principales atractivos turísticos del lugar, así como información de paquetes turísticos.

El enfoque principal de esta propuesta de sistema web son los hoteles pequeños ubicados en lugares turísticos, los cuales en la actualidad carecen de sistemas de información para el control del registro de sus reservaciones.

\section{$3 \quad$ Herramientas Existentes}

En esta sección se describen tres herramientas que permiten personalizar sitios web para hoteles con motor de reservaciones en línea: PRO Internacional [8], Creowebs [9] y Obehotel [10].

\subsection{PRO Internacional}

De acuerdo a su sitio web, PRO Internacional [8] es una empresa dedicada a brindar servicios web integrales, que van desde la creación de sitios web con alto grado de funcionalidad e interactividad, hasta acciones de compromiso en redes sociales y campañas de posicionamiento en buscadores. Entre los sitios web con alto grado de funcionalidad que crea PRO Internacional, se encuentran los sitios web para hoteles con módulo integrado de reservaciones de habitaciones en línea.

Estos sitios web cuentan con las siguientes funcionalidades: reserva de habitaciones en línea; tarifarios por temporadas; galería de fotos; suscripción a boletín electrónico; comentarios (opcional); encuestas; buzón de sugerencias.

Cabe señalar que PRO Internacional no es una herramienta gratuita, y no cuenta con demostración.

\subsection{Creowebs}

Creowebs [9] es una herramienta web que permite crear sitios web a partir de una variedad de plantillas predeterminadas, las cuales están diseñadas para diferentes tipos de sitios web, tales como blogs, sistemas de reservaciones para hoteles o restaurantes, etc. Creowebs proporciona los siguientes módulos para creación de sitios web: plantillas; importar contenido; editor de imágenes; blog y noticias; redes sociales; videos; música; galerías de fotos; formulario de contacto; compartir archivos; pagos por PayPal; Uso de Google AdSense; reservaciones; Uso de Google Maps; encuestas; visualización de ruta de navegación; versión para dispositivos móviles; subdominios;

Para conocer más sobre el funcionamiento de esta herramienta web, sus ventajas y desventajas, se creó una cuenta en el sitio web y se procedió a crear un sitio web para un 
hotel. Se realizaron diferentes pruebas para conocer a fondo cada una de sus funcionalidades; por ejemplo, se cambió la tipografía, se editó la información de ciertas secciones del sitio, se modificaron los formularios prediseñados, se dieron de alta habitaciones y temporadas, así como los precios correspondientes en cada una de ellas; y sobre todo, se realizaron consultas de disponibilidad y peticiones de reservaciones. Como ejemplo, en la Figura 1 se muestra la interfaz para registrar una nueva habitación en la herramienta Creowebs.

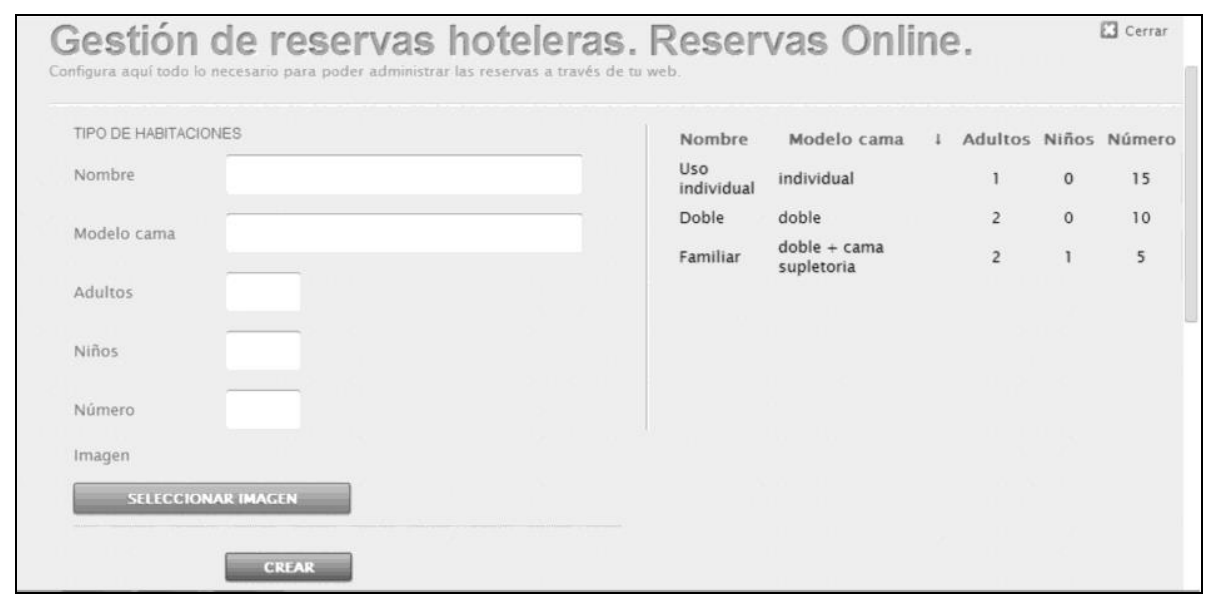

Fig. 1. Registro de nueva habitación en Creowebs.

\subsection{ObeHotel}

De acuerdo a sus creadores, ObeHotel [10] es el conjunto de las distintas soluciones, desarrollos y aplicaciones tecnológicas dirigidas a los hoteles para su comercialización y distribución en línea, orientadas a mejorar tanto los procesos de los hoteleros como la experiencia de los clientes. ObeHotel ofrece cuatro diferentes servicios: pasarela de reservaciones; web completa; channel manager; y máxima visibilidad.

El servicio de web completa de ObeHotel es el que está relacionada con esta propuesta, por lo cual fue el que se analizó. Las principales características de web completa son las siguientes: diseño personalizado; creación y configuración de cualquier tipo de habitación; reservación de múltiples habitaciones; gestión de ofertas y paquetes; posicionamiento en buscadores; diferentes tarifas para el sitio web y para los canales de venta; código de rastreo para monitorear visitas; integración con Google Analytics y Google Adwords; módulo web de opiniones de clientes.

ObeHotel ofrece un sitio web de demostración, en el que se pueden probar las funcionalidades que ofrece hacia los huéspedes potenciales. En este sitio de prueba se puede verificar disponibilidad; reservar habitaciones; conocer los tipos de habitaciones; los ser- 
vicios, paquetes y ofertas; mapa de ubicación del hotel; un formulario de contacto; tour fotográfico y un video tour. Como ejemplo, en la Figura 2 se puede observar el formulario para verificar la disponibilidad de habitaciones.

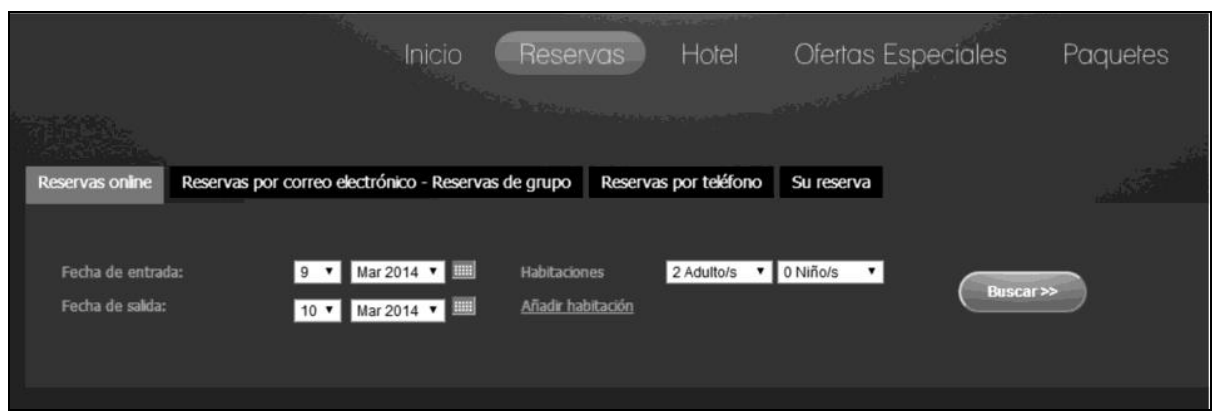

Fig. 2. Formulario de disponibilidad en ObeHotel.

Debido a que el sistema no es gratuito y no cuenta con una demostración en la que se pueda interactuar directamente con el sistema en la parte de administración, no se pudieron probar los módulos que corresponden al usuario administrador.

\subsection{Comparación}

En la Tabla 2 se muestra una comparación de funcionalidades entre las tres herramientas mencionadas anteriormente: PRO Internacional, Creowebs y ObeHotel; la paloma indica que la herramienta tiene la funcionalidad, mientras que el tache indica que no la tiene. A continuación se muestra una descripción de las características mostradas en la Tabla 2, las cuales se utilizaron para evaluar las herramientas analizadas.

Tabla 2. Características para evaluar las herramientas analizadas.

\begin{tabular}{lccc}
\hline \multicolumn{1}{c}{ Características } & PRO Internacional & Creowebs & ObeHotel \\
\hline Gestión de habitaciones & $\checkmark$ & $\checkmark$ & $\checkmark$ \\
Reservación múltiple & $\boldsymbol{x}$ & $\mathbf{x}$ & $\checkmark$ \\
Tarifas por temporadas & $\checkmark$ & $\checkmark$ & $\mathbf{x}$ \\
Gestión de ofertas y paquetes & $\mathbf{x}$ & $\checkmark$ & $\checkmark$ \\
Mapa de disponibilidad & $\checkmark$ & $\checkmark$ & $\checkmark$ \\
Comentarios & $\checkmark$ & $\checkmark$ & $\checkmark$ \\
Encuestas & $\checkmark$ & $\mathbf{x}$ & $\mathbf{x}$ \\
Buzón de sugerencias & $\checkmark$ & $\checkmark$ & $\mathbf{x}$ \\
Plantillas prediseñadas & $\checkmark$ & $\checkmark$ & $\mathbf{x}$ \\
Formularios personalizables & $\mathbf{x}$ & & $\mathbf{x}$
\end{tabular}




\begin{tabular}{llcc} 
Estadísticas & $\mathbf{x}$ & $\mathbf{x}$ & $\checkmark$ \\
Versión móvil & $\mathbf{x}$ & $\checkmark$ & $\mathbf{x}$ \\
\hline
\end{tabular}

Gestión de habitaciones. Funcionalidad para permitir al administrador la creación, modificación y eliminación de habitaciones.

Reservación múltiple. Funcionalidad para permitir la reservación múltiple en una sola transacción por el mismo huésped potencial.

Tarifas por temporadas. Es la funcionalidad para registrar tarifas por habitación según la temporada del hotel; dichas temporadas son definidas por el administrador con fechas de inicio y fin sin posibilidad de traslapes entre ellas.

Gestión de ofertas y paquetes. Funcionalidad que permite la creación, modificación y eliminación de ofertas y paquetes. Las ofertas son descuentos o promociones en el precio de los servicios; y los paquetes son un grupo de servicios en conjunto.

Mapa de disponibilidad. Esta funcionalidad permite visualizar el mapa de disponibilidad con la relación de habitaciones disponibles y ocupadas. En algunos casos se muestra gráficamente y en otros es una tabla del total de las habitaciones y su estado.

Comentarios. Funcionalidad para incluir una sección que permita a los huéspedes publicar comentarios sobre su estancia en el hotel.

Encuestas. Funcionalidad que permite al administrador del hotel realizar encuestas de satisfacción de la estancia de los huéspedes.

Buzón de sugerencias. Esta funcionalidad permite incluir un buzón de sugerencias con un formulario, donde el huésped puede emitir una sugerencia al hotel y ésta se envía al correo electrónico del administrador.

Plantillas prediseñadas. Funcionalidad que permite al administrador del hotel elegir una plantilla prediseñada para su sitio web.

Formularios personalizables. Es la funcionalidad que permite al administrador del hotel personalizar formularios; añadiendo y eliminando campos.

Estadísticas. Funcionalidad que permite al administrador conocer información relevante sobre las visitas al sitio web.

Versión móvil. Esta funcionalidad permite visualizar el sitio web desde un dispositivo móvil, ajustando el diseño del sitio web según la resolución del dispositivo.

\section{Propuesta}

En esta sección se presenta la propuesta del sistema web, la metodología a seguir, así como los avances que se han alcanzado. La propuesta es un sistema web personalizable para hoteles con motor de reservaciones, utilizando las siguientes tecnologías: Java, HTML, CSS y JavaScript para la creación del sistema web; MySQL como manejador de bases de datos relacionales; y siguiendo una metodología iterativa e incremental para el levantamiento de requerimientos, análisis y diseño del sistema. 
El administrador podrá personalizar el sistema web según sean las características de su hotel: habitaciones, precios por temporada, servicios, paquetes, promociones, información de contacto, atracciones, así como gestionar las reservaciones. El huésped potencial podrá consultar la información completa del hotel; servicios, promociones, paquetes, atracciones, información de contacto, así como realizar su reservación. La interfaz del huésped potencial se diseñará utilizando el patrón de diseño web adaptable (Responsive Web Design), para adaptar la interfaz según el dispositivo que utilice el usuario para acceder al sitio web, como se muestra en la Figura 3.

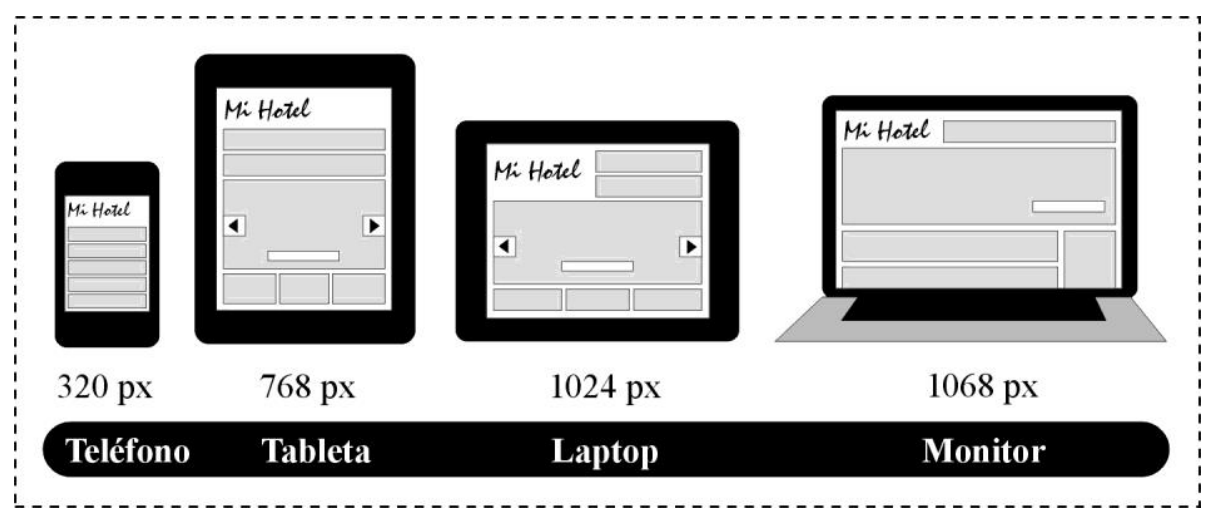

Fig. 3. Diseño web adaptable.

\subsection{Metodología}

Para la creación del sistema web se plantearon las siguientes actividades.

Identificar usuarios. En esta etapa se realizarán entrevistas a administradores de hoteles con el objetivo de conocer las actividades que realizan para la administración del hotel y las características de éste. También se realizarán entrevistas a huéspedes potenciales para conocer los medios de búsqueda y reservación que utilizan, así como sus preferencias al viajar. Se analizarán los resultados y se definirán dos perfiles de usuario: administrador y huésped potencial; así como las características del hotel.

Realizar levantamiento de requerimientos. En esta etapa se elegirá a un hotel para realizar el levantamiento de requerimientos, con el objetivo de adquirir la información necesaria para desarrollar el sistema web.

Diseñar e implementar un módulo para administración del hotel. En esta etapa se diseñará e implementará un módulo que permita la administración de las reservaciones. Las funciones para llevar a cabo la administración de reservaciones del hotel son el mapa de disponibilidad y reservaciones, entre otras.

Diseñar e implementar un módulo que permita la personalización del portal web. En esta etapa se diseñará e implementará un módulo que permita al administrador del 
hotel personalizar el portal web con las características particulares del hotel. Se permitirá la creación, modificación y eliminación de habitaciones, temporadas, paquetes y promociones; así como encuestas y formularios.

Diseñar e implementar un módulo para reservaciones en línea. En esta etapa se diseñará e implementará un módulo que permita reservar una o varias habitaciones por medio del portal web; así como el envío automático de un correo electrónico al usuario que reservó, con los datos de su reservación y la confirmación de la misma.

\subsection{Avances de la Propuesta}

Dentro de los avances de esta propuesta, ya se identificaron a los dos principales usuarios del sistema web propuesto, a través de entrevistas: administradores y huéspedes potenciales. También se realizó un primer levantamiento de requerimientos con el administrador de un hotel; análisis y diseño con diagramas de clases, de colaboración y de secuencia; así como un mapa de navegación y un conjunto de interfaces que ya han sido validadas con el usuario administrador. En la Figura 4 se muestra el mapa de navegación del sistema, con el administrador, y el huésped potencial.

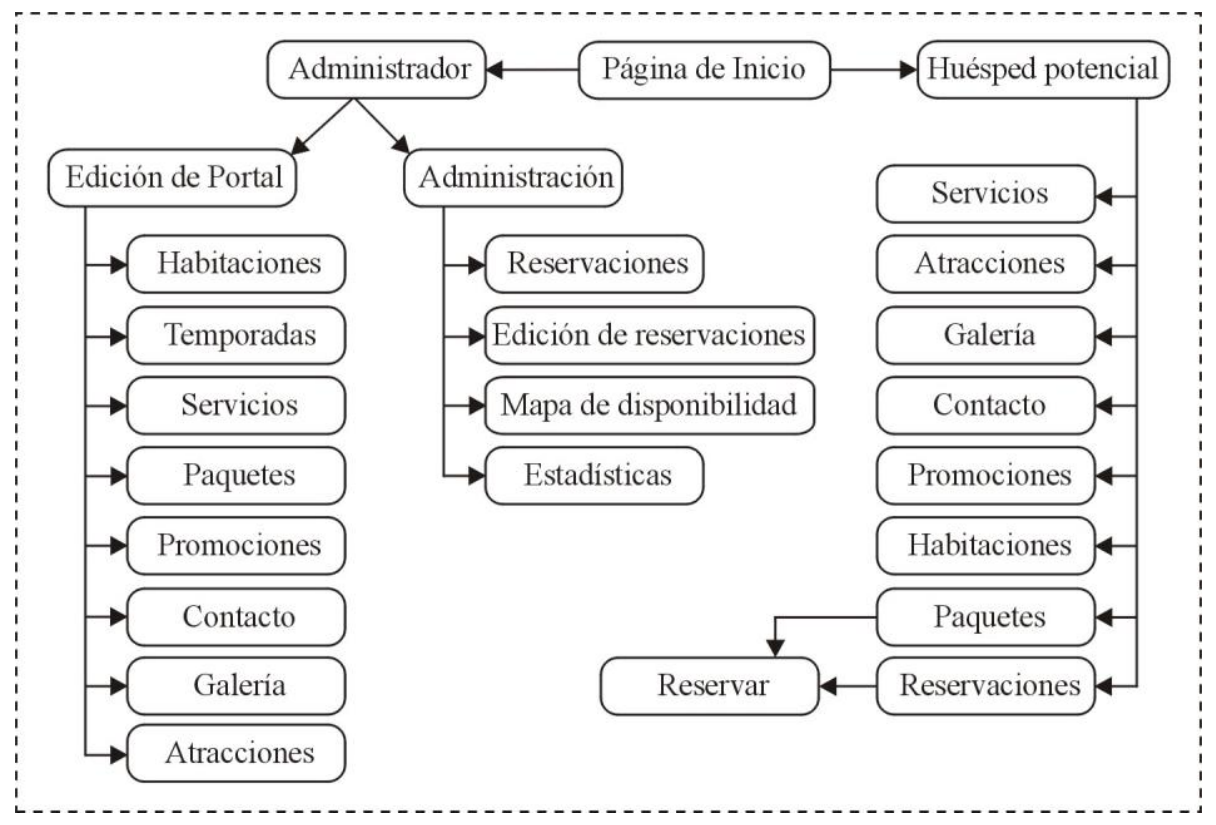

Fig. 4. Mapa de navegación. 
El mapa de navegación muestra el conjunto de páginas web a las que tiene acceso cada usuario del sistema web. El administrador puede acceder a dos secciones principalmente: la edición del portal, donde puede realizar altas, bajas y cambios de habitaciones, temporadas, servicios, paquetes, promociones, contacto, galería, y atracciones; y la administración, la cual incluye el registro y edición de reservaciones, mapa de disponibilidad y estadísticas. El huésped potencial puede acceder a las siguientes páginas web: servicios, atracciones, galería, contacto, promociones, habitaciones, paquetes, y puede realizar reservaciones de habitaciones y paquetes.

Como ejemplo, en la Figura 5 se muestra la interfaz gráfica del mapa de disponibilidad de las habitaciones de un hotel, dentro del sistema web. Cabe señalar que las interfaces gráficas desarrolladas son una versión preliminar; falta incorporar las plantillas personalizables, las cuales proporcionarán creativos diseños para la visualización del sistema web en computadoras de escritorio, laptops, y en dispositivos móviles como tabletas y teléfonos inteligentes.

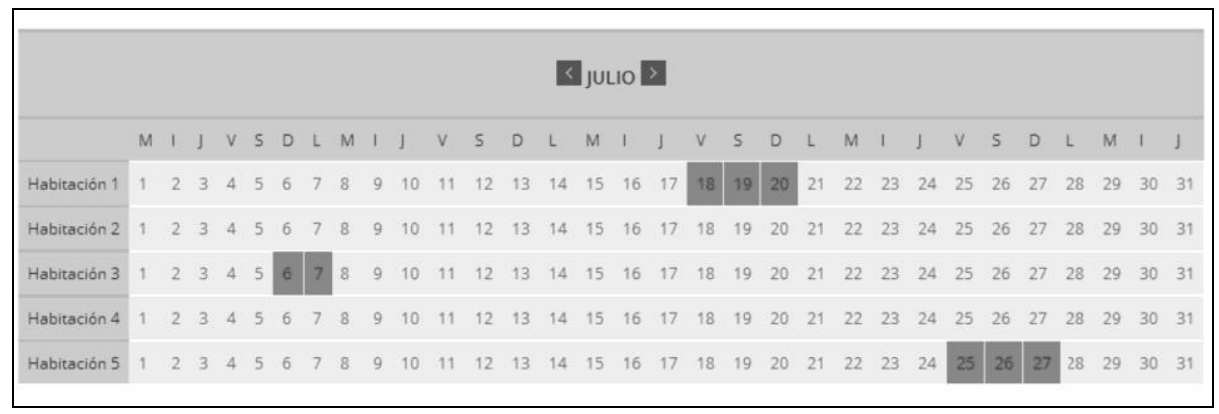

Fig. 5. Mapa de disponibilidad del sistema web.

\section{Conclusiones y Trabajo Futuro}

El propósito de este trabajo es generar un sistema web personalizable para facilitar a administradores de hoteles pequeños el control de reservaciones de sus habitaciones, gestionando las ventas desde su propio sitio web; así como para facilitar y garantizar de inmediato las reservaciones para sus huéspedes potenciales. Adicionalmente, el sistema web será visualizado automáticamente en diferentes dispositivos, tales como computadoras de escritorio, laptops, tabletas y teléfonos inteligentes, siguiendo el patrón de diseño web adaptable.

En el artículo se presentó un análisis de herramientas existentes similares, y se proporcionó una comparativa de funcionalidades. Dentro del avance de la propuesta presentada, ya se han identificado a los dos usuarios de este sistema web, y se han creado sus perfiles: administrador y huésped potencial. También se ha realizado un primer levantamiento de 
requerimientos con un administrador de un hotel; análisis y diseño con sus correspondientes diagramas de clases, de colaboración y de secuencia; así como un mapa de navegación y un conjunto de interfaces gráficas que ya han sido validadas con el usuario administrador.

Dentro del trabajo futuro, es necesario completar el diseño e implementación del sistema web, al mismo tiempo de mantener una estrecha comunicación con el administrador del hotel para su validación. También es necesario realizar pruebas de funcionalidad y de usabilidad, tanto con administradores de hoteles, como con huéspedes potenciales. Finalmente, se realizarán ajustes al sistema después de las pruebas realizadas, y se pondrá en funcionamiento en los hoteles para los cuales se está diseñando.

\section{Referencias}

1. Trip Barometer: Encuesta realizada a hoteleros y viajeros. Disponible en: http://www.tecnohotelnews.com/wp-content/uploads/2013/03/Infografia_TripBarometer.jpg. Último acceso en Junio de (2014)

2. Sitio web del hotel Las cúpulas. Disponible en: http://lascupulas.com.mx/. Último acceso en Junio (2014)

3. Sitio web del hotel La casa del laurel. Disponible en: http://www.hotelentaxco.com/es/index.html Último acceso en Junio (2014)

4. Sitio web del hotel El encanto. Disponible en: http://elencantobernal.com/acerca. Último acceso en Junio (2014)

5. Sitio web del hotel Hotel Malinalco. Disponible en: http://www.hotelmalinalco.com.mx/. Último acceso en Junio (2014)

6. Sitio web del hotel Casa abierta. Disponible en: http://www.casabierta.com.mx/index.html. Último acceso en Junio (2014)

7. Sitio web del hotel Hotel Cristal. Disponible en: http://www.hotelcristalchignahuapan.com.mx/. Último acceso en Junio (2014)

8. Sitio web de PRO Internacional. Disponible en: http://www.prointernacional.com/. Último acceso en Junio de (2014)

9. Sitio web de Creowebs. Disponible en: https://creowebs.com/. Último acceso en Junio (2014)

10. Sitio web de ObeHotel. Disponible en: http://www.obehotel.com/. Último acceso en Junio (2014)

11. Tendencias en diseño web. Disponible en: http://www.tecnohotelnews.com/2013/07/lastendencias-de-futuro-en-diseno-web-para-hoteles/. Último acceso en Junio (2014)

12. Responsive Web Design. Disponible en: http://www.designtribe.ie/news/24/61/NewResponsive-B-B-Hotel-website. Último acceso en Junio (2014)

13. Motores de reservaciones. Disponible en: http://www.tecnohotelnews.com/2013/05/motores-de-reservas-flexibles-e-integrados/. Último acceso en Junio (2014)

14. Infografía. Disponible en: http://www.alojapro.com/blog/wp-content/uploads/2014/01/infografia.jpg. Último acceso en Junio (2014) 